\title{
Single-base-resolution methylomes of populus trichocarpa reveal the association between DNA methylation and drought stress
}

\author{
Dan Liang ${ }^{1 \dagger}$, Zhoujia Zhang ${ }^{1 \dagger}$, Honglong $\mathrm{Wu}^{3+}$, Chunyu Huang ${ }^{2 \dagger}$, Peng Shuai ${ }^{1 \dagger}$, Chu-Yu Ye ${ }^{1}$, Sha Tang ${ }^{1}$, \\ Yunjie Wang ${ }^{2}$, Ling Yang ${ }^{3}$, Jun Wang ${ }^{2}$, Weilun Yin ${ }^{1 *}$, Xinli Xia ${ }^{1 *}$ \\ From International Symposium on Quantitative Genetics and Genomics of Woody Plants \\ Nantong, China. 16-18 August 2013
}

\begin{abstract}
Background: DNA methylation is an important biological form of epigenetic modification, playing key roles in plant development and environmental responses.

Results: In this study, we examined single-base resolution methylomes of Populus under control and drought stress conditions using high-throughput bisulfite sequencing for the first time. Our data showed methylation levels of methylated cytosines, upstream $2 \mathrm{kp}$, downstream $2 \mathrm{~kb}$, and repeatitive sequences significantly increased after drought treatment in Populus. Interestingly, methylation in $100 \mathrm{bp}$ upstream of the transcriptional start site (TSS) repressed gene expression, while methylations in 100-2000bp upstream of TSS and within the gene body were positively associated with gene expression. Integrated with the transcriptomic data, we found that all cis-splicing genes were non-methylated, suggesting that DNA methylation may not associate with cis-splicing. However, our results showed that $80 \%$ of trans-splicing genes were methylated. Moreover, we found 1156 transcription factors (TFs) with reduced methylation and expression levels and 690 TFs with increased methylation and expression levels after drought treatment. These TFs may play important roles in Populus drought stress responses through the changes of DNA methylation.
\end{abstract}

Conclusions: These findings may provide valuable new insight into our understanding of the interaction between gene expression and methylation of drought responses in Populus.

\section{Background}

Populus (Populus sp.) is an ideal model system for investigating molecular mechanisms of trees in response to environmental stresses, due to its advantages including rapid growth, high yield, easy propagation, importance in the economy and available genomic resources $[1,2]$. Drought is a major abiotic stress that limits the survival and growth of young poplar plants [3]. Therefore, many

\footnotetext{
*Correspondence: xiax@@bjfu.edu.cn; yinwl@bjfu.edu.cn

† Contributed equally

'College of Biological Sciences and Technology, National Engineering Laboratory for Tree Breeding, Beijing Forestry University, Beijing 100083, China

Full list of author information is available at the end of the article
}

studies have focused on understanding drought responsive mechanisms in Populus [4-6].

DNA methylation is an important biological form of epigenetic modification. Currently, methylation analysis is widely used to explore various mechanisms underlying biological survival in plants $[7,8]$, humans [9], and insects [10]. Plant genomes show extensive cytosine methylation at CG, CNG (N represents any nucleotide), and $\mathrm{CHH}(\mathrm{H}$ represents $\mathrm{A}, \mathrm{C}$ or $\mathrm{T}$ ) sites [11]. Previous studies indicated that the effect of DNA methylation in plants refers to DNA methylation preventing DNA transcription by combining with the genomic sequence of transcription factors, and another refers to specific proteins (known as methyl-CpG binding proteins) binding with methylated DNA and acting as competitors of transcription factors. 
Complexes of proteins affect chromosome histone acetylation, leading to transcriptional inhibition $[12,13]$. Methylation induced by biotic stress is generally associated with the silencing of parasitic DNA and expression of resistant genes, while abiotic stress-induced methylation is supposed to be linked with the transcription factors which participate in numerous biochemical pathways involved in acclimatization and stress response in plants [14].

Numerous studies have shown that DNA methylation levels could be affected by plant stress in Arabidopsis, rice, pea and other plants [15-18], but few about trees. Uthup et al. have reported the identification of DNA methylation patterns and their putative relationship with abiotic stress in the tree crop Hevea brasiliensis [14]. The percentage of hypermethylated loci increased, and that of fully methylated loci clearly decreased in Quercus ilex trees exposed to drought [19]. However, no studies performed methylation analysis of Populus at the genomic level under drought stress by using high-throughput bisulfite sequencing. To improve our understanding of the resistance mechanisms at the molecular level in Populus, we investigated DNA methylomes of Populus trichocarpa under normal and drought conditions, focusing on epigenetic regulation of stress responses.

\section{Results}

Bisulfite sequencing of the Populus trichocarpa genome

To generate the single-base resolution methylome of P. trichocarpa under normal (i.e., well water; WW) and drought stress (i.e., water stress; WS) conditions, we applied the Illumina Hiseq 2000 platform for bisulfite sequencing of DNA extracted from leaves. Reads that aligned to the unmethylated lambda DNA, which were added to the total DNA before applying the bisulfite treatment, were used to calculate the conversion rate. The conversion rates of WW and WS were $99.40 \%$ and $99.45 \%$, respectively. Two sets of raw data were obtained from sequencing, with the output of 16.86 giga base pair (Gb) in WW sample and $16.63 \mathrm{~Gb}$ in WS. We subsequently used a series of filter criteria to ensure the data quality, including trimming low-quality reads(reads that contain more than 50\% unknown bases) and retaining unique-aligned reads. Finally, $72.96 \%$ of the reads in WW and $76.69 \%$ of the reads in WS were used for further analysis. These data were estimated to cover the whole genome with 28.45and 29.93-fold sequencing depths (Additional file 1). To avoid the effects of individual SNPs, the online original reference (http://genome.jgi-psf.org/poplar/poplar.home. html) was modified by the resequence data.

\section{DNA methylation in Populus trichocarpa}

Our results showed that methylated cytosines (mCs) accounting for $10.04 \%$ of all cytosines in the whole genome under drought stress were significantly more than those $(7.75 \%)$ in WW (Additional file 2). Distribution of cytosine methylation levels showed that more proportions of $\mathrm{mCG}$ and $\mathrm{mCHG}$ have higher methylation levels as compared with $\mathrm{mCHH}$ (Figure 1-a). CG sites were much higher methylated in gene region than other sites (Figure 1-b). Additionally, our results showed that methylated sites were concentrated in the non-CG sites in the Populus genome, especially in $\mathrm{mCHH}$ (Figure 1-c), and the basic group of $\mathrm{HH}$ or $\mathrm{mCHH}$ tended to contain twofold more A or T (Additional file 3). Since the chromosome structure of the $P$. trichocarpa genome is imperfect, we concatenated the remaining fragments as the $20^{\text {th }}$ scaffold in addition to the existing 19 scaffolds and then analyzed the distribution of the mCs across chromosomes, which shows that there was a high methylated cytosine density in centromere regions that consisted of more repetitive sequences (Additional file 4).

The following analysis was to investigate the methylation profiles of coding sequences (CDSs), introns, untranslated regions (UTRs), small RNAs, repetitive DNAs under relative and absolute methylation standards [10]. We found that methylation levels in coding regions were higher than that in 5'- and 3'-UTRs. The methylation levels of repetitive regions were significantly higher than those of gene body regions $(\mathrm{p}<7.71 \mathrm{E}-07$, Wilcoxon RankSum test). Furthermore, the methylation levels of 2000bp upstream of the transcriptional start site (up2K), $2000 \mathrm{bp}$ downstream of the transcriptional termination site (down2K), and repetitive sequences were significantly higher after drought treatment $(\mathrm{p}<0.001625$, Wilcoxon RankSum test) (Figure 2).

Gene Ontology analysis demonstrated biological processes related to biological regulation were enriched in both newly methylated and demethylated genes after drought stress as compared with the whole Populus genome, indicating that methylation or demethylation of these biological regulation related genes may play important roles in drought stress response of Populus (Additional file 5-a,b).

\section{Prediction and validation of splicing events in P. trichocarpa}

We used transcriptome sequencing technology to further investigate the expression profiles of two samples (i.e., WW and WS). A total of 130,884,860 and 138,441,434 raw reads were generated, and 86,385,459 (66.00\%) and 90,236,283 (65.18\%) unique alignment reads were retained in WW and WS, respectively (Additional file 6). We analyzed two splicing forms of the genes, i.e., the trans-splicing and cis-splicing models. Cis-splicing occurs within a single transcript, which can produce multiple mRNA transcripts. Trans-splicing occurs at the bonding point, which is formed by two different genes [26]. 


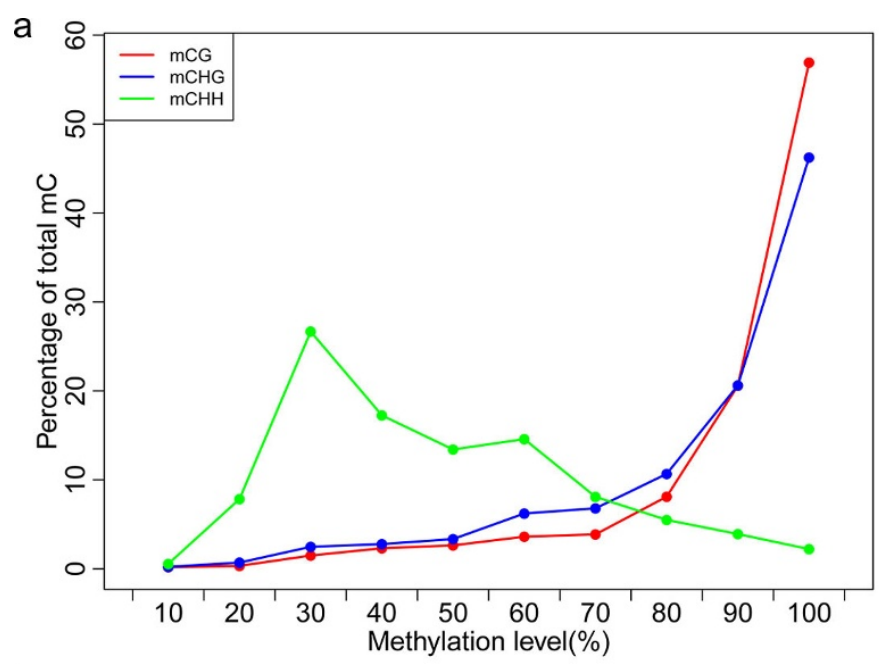

b
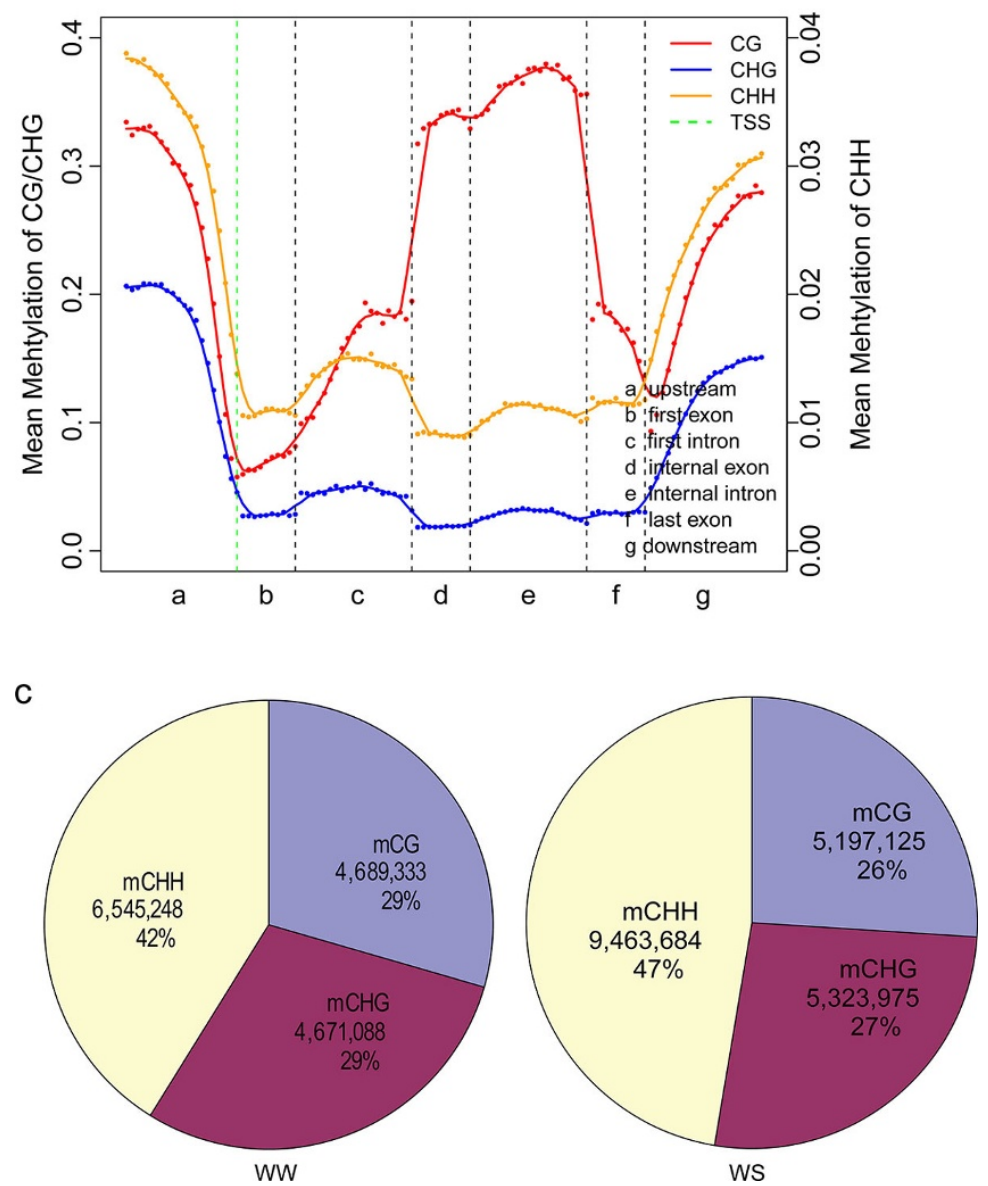

Figure 1 The global pattern of Populus DNA methylomes. (a) The percentage of methylated cytosines distribution in each sequence context $(\mathrm{H}=\mathrm{A}, \mathrm{T}, \mathrm{C})$. The $y$ axis indicates the percentage methylated cytosines according to each methylation level which show on $x$ axis.(methylation level = \# of $\mathrm{mC} /$ \# of C* 100\%) (b) Distribution of CG, CHG and CHH methylation levels in each sequence context of gene related region, including upstream, first exon, first intron, internal exon, internal intron, last exon and downstream.. The $x$ axis shows seven elements, the $y$ axis represents average methylation levels of cytosines. The red dots represent average methylation levels of bins, the curve shows the average value of 5 bins with 1-bin step, and the green dotted line indicate TSSs (transcription start sites) (c) The percentage and absolute number of methylated cytosines that identified in WW(left) and WS(right) in each sequence context. 
Recently, alternative splicing and gene fusion were discovered by high-throughput sequencing in more species, such as human [27], rice [24] and Arabidopsis [28]. However, no information on alternative splicing in Populus was available. We found four alternative splicing types in P. trichocarpa: A) Exon skipping, B) intron retention, $C$ ) alternative 5 'splice sites, and D) alternative 3 ' splice sites, according to the classification of alternative splicing types in rice [24] and 11791 and 13251 alternative splicing genes in WW and WS, respectively. At least $20 \%$ of intron-containing genes in P. trichocarpa were spliced, which is lower than that (42\%) in Arabidopsis [28]. More genes in WS were alternatively spliced in four alternative splicing types (Figure 3), especially the alternative 3' splice sites were more increased, suggesting that alternative splicing can be regulated and associated with environmental stress as previous reports $[29,30]$ and methylation may play more acting on alternative 3' splice sites after drought treatment in Populus. Particularly, in terms of the methylation level of 20 bp sequences containing alternative splicing sites, more than $80 \%$ of all alternative splicing sites in two samples were non-methylated, while none of the genes were methylated (Additional file $7-a)$. Interestingly, we found that $80 \%$ of the fusion genes were methylated and the splicing events were decreased after the drought treatment (Table 1). To verify the accuracy of the fusion gene, that identified 227 and 161 in WW and WS, we randomly selected 10 genes in the two groups of fusion materials to test by RT-PCR, six of which were verified (Additional file 7-b). The probability $(6 / 10)$ was slightly lower than expected based on the accuracies in human and rice [24,27].

\section{Effects of DNA methylation on gene expression in P. trichocarpa}

To compare the effects of Populus DNA methylation on gene expression in two samples (i.e., WW and WS), we used transcriptome sequencing to determine the expression levels of methylated and unmethylated genes. Generally speaking, the expression levels of examined 17714 expressed genes were positively correlated with their methylation levels. We found that 7392 genes exhibited rising trends in methylation and expression levels after drought treatment. Methylation and expression levels were both reduced in 10322 genes under drought stress.

To further analyze the relationship between gene expression and methylation levels, we divided these genes into four categories based on expression levels: High expression, medium expression, low expression, and silent genes, from the bottom one-third to the top one-third (Figure 4-a). We found that silent genes had significantly higher methylation levels than expressed genes, indicating that gene silence may be caused by high methylation level ( $p=6.8 \mathrm{E}-08$, Wilcoxon Rank Sum test). For expressed genes, methylation levels in gene body and upstream region were positively correlated with gene expression (Additional file 8). Nonetheless, gene expression levels had negative correlations with methylation at transcription start sites (TSSs), transcriptional termination regions (TTRs) and downstream regions.

Gene-body methylation genes had significantly higher expression levels than body-unmethylated genes ( $p=$ 6.01E-07, Wilcoxon rank sum test) indicating a positive correlation between gene expression and gene-body methylation (Figure 4-c). Interestingly, the results showed that upstream2k-methylated genes have significantly higher expression level than upstream $2 \mathrm{k}$ unmethylated genes ( $p=7.41 \mathrm{E}-05$, Wilcoxon rank sum test) [31] (Figure 4-d). However, further analysis was performed on the 100bp upstream of the transcriptional start site (TSS), and found that genes methylated in this region had significantly higher expression level than unmethylated genes ( $p=8.6 \mathrm{E}-06$, Wilcoxon rank sum test), indicating that methylation of this region induced gene expression (Figure 4-e). Coincident with the situation of $100 \mathrm{bp}$ upstream of the TSS, downstream $2 \mathrm{k}$ unmethylated genes have higher expression level than downstream2k-methylated genes (Figure 4-f).

As regards changes after drought treatment, the methylation levels of the silent genes increased significantly ( $p=6.796 \mathrm{E}-08$, Wilcoxon rank sum test) (Figure 4-a,b). The expression level of upstream $2 \mathrm{k}$-unmethylated genes was reduced, while it increased for genes methylated in 100bp upstream of TSS. Downstream $2 \mathrm{k}$-unmethylated genes have higher expression level after treatment. Genebody methylated and unmethylated genes have no significant change.

\section{Differentially expressed and methylated transcription factors}

Due to critical roles of transcription factors (TFs) in responses to external stimuli by influencing the expression of downstream targets, we identified differentially

Table 1 Numbers of methylated and unmethylated gene fusions.

\begin{tabular}{|c|c|c|c|c|c|}
\hline Sample & $\begin{array}{c}\text { Methylated genes in the } \\
\text { genome }\end{array}$ & $\begin{array}{l}\text { Unmethylated genes in } \\
\text { the genome }\end{array}$ & $\begin{array}{l}\text { Fusion genes both } \\
\text { methylated }\end{array}$ & $\begin{array}{l}\text { Fusion genes both } \\
\text { unmethylated }\end{array}$ & $\begin{array}{c}\text { Fusion genes one } \\
\text { methylated }\end{array}$ \\
\hline WW & 23983 & 8071 & 194 & 4 & 29 \\
\hline WS & 22498 & 8895 & 130 & 11 & 20 \\
\hline
\end{tabular}


expressed and methylated TFs in $P$. trichocarpa according to two TF databases (i.e., DPTF (http://dptf.cbi.pku. edu.cn/index.php) [32] and PlnTFDB (http://plntfdb.bio. uni-potsdam.de/v3.0/) [33]. A total of 1156 TFs showed reduced methylation and expression levels after drought treatment. These TFs were distributed in 79 families, including MYB, AP2, WRKY, NAC, and bHLH. We also found that 690 TFs showed increased methylation and expression levels after drought treatment. Most of them belong to $\mathrm{C} 3 \mathrm{H}, \mathrm{PHD}, \mathrm{MYB}, \mathrm{ARF}$, and bZIP families (Additional file 9).

Transposable elements (TEs) can influence gene regulation on a genomic scale by carrying potential transcription-regulating signals. To further analyze the mechanism underlying the TF response to drought stress by hypermethylation of transposons, we found 389 and 334 TFs were located by TEs in promoters and in gene bodies using the described method by Thornburg [34], respectively. The methylation levels of 138 TEs in promoters were elevated by drought treatment, while those of 251 TEs were reduced. The methylation levels of 163 TEs in gene body regions were elevated under drought treatment, while those of 171 TEs were reduced. These two kind of transcription factors were divided into 64 and 60 families (Additional file 10, 11), respectively, which were concentrated in both the major transcription factor families and some related to stress signal transduction, such as $\mathrm{C} 2 \mathrm{C} 2$ and EIL proteins.

\section{Disscussion}

Although the relationship between DNA methylation and gene expression has been explored in Populus and other plants $[35,36]$, the resolution of genome-wide methylated cytosines requires more elaborate and comprehensive methylomic studies to characterize the functional effects of Populus DNA methylation. We obtained the single-base resolution methylomes of Populus, which was used to investigate the changes of DNA methylation under drought treatment via the high-throughput sequencing, and found the genome-wide methylation level in $P$. trichocarpa was slightly higher than that in Arabidopsis [24] but lower than humans [9]. Moreover, having found plausible patterns with our comprehensive dataset, our results have a number of implications, which will have a promising application in the future research on Populus and may give some cue on other plants' studies.

\section{The relationship of methylation and splicing events in P. trichocarpa}

The role of DNA methylation in alternative splicing is supported by Shukla et al. [37] in CD45 cells, as well as by Chodavarapu et al. in Arabidopsis thaliana, showing that DNA methylation is highly enriched in exons and may have an important role in alternative splicing [38]. We did not find any methylated sites in more than $80 \%$ of alternative splicing genes. In contrast with the previous results, DNA methylation is not enriched in exons and the methylation levels in exons were not significant higher than the other regions in Populus. Therefore, our results can't speculate that DNA methylation may play an important role in alternative splicing in Populus genome. By contrast, our results showed that all fusion genes were methylated, that inferred that this phenomenon might be associated with the mechanisms of two different splicing forms. Methylation may not affect the enzymatic reaction that results in alternative splicing, but can affect chromosomal rearrangements, RNA editing and other structural variations what might cause the occurrence of gene fusion.

Drought treatment has different effects on two different splicing forms, the number of genes with alternative splicing events was increased, while fusion genes were reduced after the drought treatment. Since the effects of drought on the complex network of signaling pathway in Populus, we can not point out the direct cause, but it can be speculated that it may be related to the formation or activity of cleavage enzyme, which main activates at 3 ' and 5 ' two positions.

\section{DNA methylation and gene expression}

The way of the expression of genes affected by DNA methylation in plant is combining with specific protein, which competes with transcription factors. The complex causes the changes in chromosome histone acetylation, leading to the inhibition of transcription, that point was more prominent in poplar. In Populus, about 60\% of silent genes were affected by methylation. After the drought treatment, the probability was increased, indicating that the influence of gene expression by methylation increased with the external environmental stimuli.

For expressed genes, methylation in $100 \mathrm{bp}$ upstream of the TSS represses gene expression, which is consistent with the findings from $A$. thaliana [39], human [9] and rice [40], confirming that neighbouring upstream of TSS methylation is a general mechanism suppressing gene expression in eukaryotes. However, we found that methylation level in 100-2000 bp upstream was positively correlated with gene expression. It is interesting that the expression of genes methylated in $100 \mathrm{bp}$ upstream of TSS were affected after the drought treatment, which indicated that drought treatment reduced the chances of combining with specific protein and increased levels of gene expression. In the TTR (transcriptional termination regions) and the downstream region, methylation and expression were negatively correlated, indicating that they had significant effect on gene expression through interfering with transcriptional termination site. 
Drought is one of the major environmental factors that affected gene expression by complex signaling networks, including cytosine methylation, histone acetylation and H3K9 methylation [17]. In Populus, the expression of gene was affected by drought on genetic elements in upstream and downstream. Combined with changes in methylation levels, $100 \mathrm{bp}$ upstream of the TSS was focused. To further understand the relationship between methylation and drought, we need to make sure the genetic elements in different locations of upstream.

\section{Methylated transcription factors with TE in drought tolerance}

Transcription factors involved in signal transduction related to various stresses, such as drought. Diverse biological activities were regulated directly or indirectly by these transcription factors. The demethylation of transcription factors under drought stress may reduce the stability of the gene, thereby affecting its expression. (For example, BZIP, WRKY, AP2 / EREBP and MYB, four major transcription factor families, play an important role in plant stress resistance, and many TFs of expression changed by methylation in our results also belong to them).

The use of transposable elements (TEs) for reverse transcription, DNA cutting and ligation or DNA binding are well-documented [41]. Changes in transposition activity correlated with methylation were first described in maize $[42,43]$. Subsequently, in both plants and animals [44], the role of DNA methylation in transposons was directly tested by loss of DNA methylation (which is sufficient for the mobilization of transposons) [45]. TEs can influence gene regulation on a genomic scale by carrying potential transcription-regulating signals. When inserted in promoter regions, they can alter gene expression patterns by introducing new transcription factor binding sites [46]. Our data indicated that transcription factors that affect gene expression after drought treatment were affected by methylated transposons in Populus. We found methylated transposons in $\mathrm{C} 2 \mathrm{C} 2$, WRKY MYB and other families that involved in signal transduction pathway of drought. When the environment changes, transposition frequency in plants increased. TEs were inserted into positive transcription factors and promoted expression, consequently increasing the expression of resistance genes. Insertion in some locations could inhibit the expression of positive transcription factors and decrease the resistance of a gene (such as by insertion of a negative transcription factor), which may have the opposite effect. Analysis of methylated transposons in transcription factors may increase our understanding of the specific mechanisms by which transcription factors regulate the stress response in plants.

\section{Conclusions}

In this study, genome-wide DNA methylation sequencing of poplar leaves with WW and WS treatments were established using high-throughput technology. It confirmed that cis-splicing sites are unmethylated, while trans-splicing sites are methylated in poplar, to our knowledge this is the first report of an association between methylation and variable splicing. It further demonstrated that DNA methylation in the regulation of stress-responsive genes by identifying methylated transposable elements (TEs) in promoters and the gene body of transcription factors. Finally, the mechanism that the DNA methylation played on the gene expression, alternative splicing, and other phenomenon was still unclear based on current technology and experiment condition, otherwise our study pave the way for future discovering of methylation-associated mechanism in large-scale integrative multi-omics datasets.

\section{Methods}

\section{Plant materials}

Populus trichocarpa (Torr. \& Gray) seedlings were planted separately into $10-\mathrm{L}$ plastic pots filled with a mixture of clay, silt and sand $(2: 2: 2, \mathrm{v} / \mathrm{v})$. They were grown under well-watered (WW) conditions in a greenhouse at Beijing Forestry University for 2 months. The plants were supplemented with light for $15 \mathrm{~h} \mathrm{~d}^{-1}$, and temperature and humidity were kept constant. According to previous reports [20-22], the plantlets were divided into two groups, which were subjected to the following watering regimes: well-watered (WW) treatment (maintaining $100 \%$ of field capacity) and water-stress (WS) treatment (maintaining 25\% of field capacity). Volumetric soil water content was kept at $24 \%$ in the WW treatment, while in the WS treatment the volumetric soil water content was kept at approximately $10 \%$. Net photosynthetic rate, transpiration rate and leaf water potential (WP) were measured using the PsyPro WP data logger (Wescor) and Li6400 Photosynthesis System (Li-Cor). After 5 weeks, plants of similar height $(\sim 75 \mathrm{~cm})$ were selected for the experiment. Five replicates (four plantlets per replicate) were used to minimize random errors. Mature leaves from the same position (8-10th counting from the apex) of different individual plants were collected and frozen immediately in liquid nitrogen for DNA extraction.

\section{BS-Seq libraries construction and sequencing}

Total DNA was extracted from the leaves collected from our experiment. Total DNA was prepared by proteinase $\mathrm{K} /$ phenol extraction following the manufacturer's instructions. The DNA was fragmented by sonication using a Bioruptor (Diagenode, Belgium) to a mean size of $\sim 250 \mathrm{bp}$, followed by the blunt ending, dA addition 
to 3'-end, and adaptor addition following the manufacturer's protocol. The bisulfite conversion of the adaptoradded DNA was performed as described previously [23]. Ultra-high-throughput pair-end sequencing was performed using the Hiseq 2000 according to the manufacturer's instructions. Raw data were processed using Illumina base-calling pipeline.

\section{Mapping and processing of BS-Seq reads}

Integrated with the Populus reference (v2.0) and resequencing data, we constructed a consensus sequence as a reference to align the methylation data. Because the strand-specific of methylation, two artificial genomes were construct, the $\mathrm{T}$ genome that whose cytosines had been converted to thymines and the A genome whose guanines had been converted to adenosines. After removing the low quality reads, all cytosines of reads 1 were converted to thymines and all guanines of reads 2 were converted to adenosines, and then all these reads were aligned to the $\mathrm{T}$ genome and $\mathrm{A}$ genome using SOAPaligner (v2.01) software with parameters -m $175-\mathrm{x} 325-\mathrm{v}$ (default 2), respectively. To increase the accuracy of methylation related analysis, reads that aligned to more than one positions were discarded.

All the unmethylated cytosine can be converted from cytosine to thymine during the process of bisulfite conversion, but the methylated cytosine will be the same. To remove the methylcytosines from the background noise which cause by the non-conversion of bisulfite, we use the conversion rate of lambda DNA input as a negative control was calculated following the formula: $\mathrm{p}=1$ (\# of methylated cytosines) / (\# of cytosines). Using this value as a measure of the false $\mathrm{mC}$ discovery rate, $\mathrm{mC}$ was identified at each base position according to the binomial probability distribution, following the correction algorithm of Lister et al. [9].

\section{Transcriptome sequencing}

For the synthesis of cDNA and Solexa sequencing, we prepared $45 \mu \mathrm{g}$ of total RNA for treated and control sample at concentrations of approximately $1500 \mathrm{ng} / \mu \mathrm{l}$. We then enriched the poly (A) mRNA using beads with Oligo (dT) and interrupted mRNA into short fragments with fragmentation buffer. Using these short fragments as templates, we synthesized first-strand cDNA with hexamer-primers and reverse transcriptase (Invitrogen). The second-strand cDNA was synthesized using buffer, dNTPs, RNaseH (Invitrogen) and DNA polymerase I (New England BioLabs). The short fragments were then purified using a QiaQuick PCR extraction kit and re- solved with EB buffer to finish the end reparation, and was connected using sequencing adaptors. After resolution by agarose gel electrophoresis, we selected fragments suitable for PCR amplification. We then constructed two paired-end libraries which were sequenced using an Illumina HiSeq 2000.

\section{Prediction of splicing events}

The pair-end reads that aligned to two different genes were considered candidate fusion genes. To ensure the accuracy of detecting the fusion genes, PE reads that

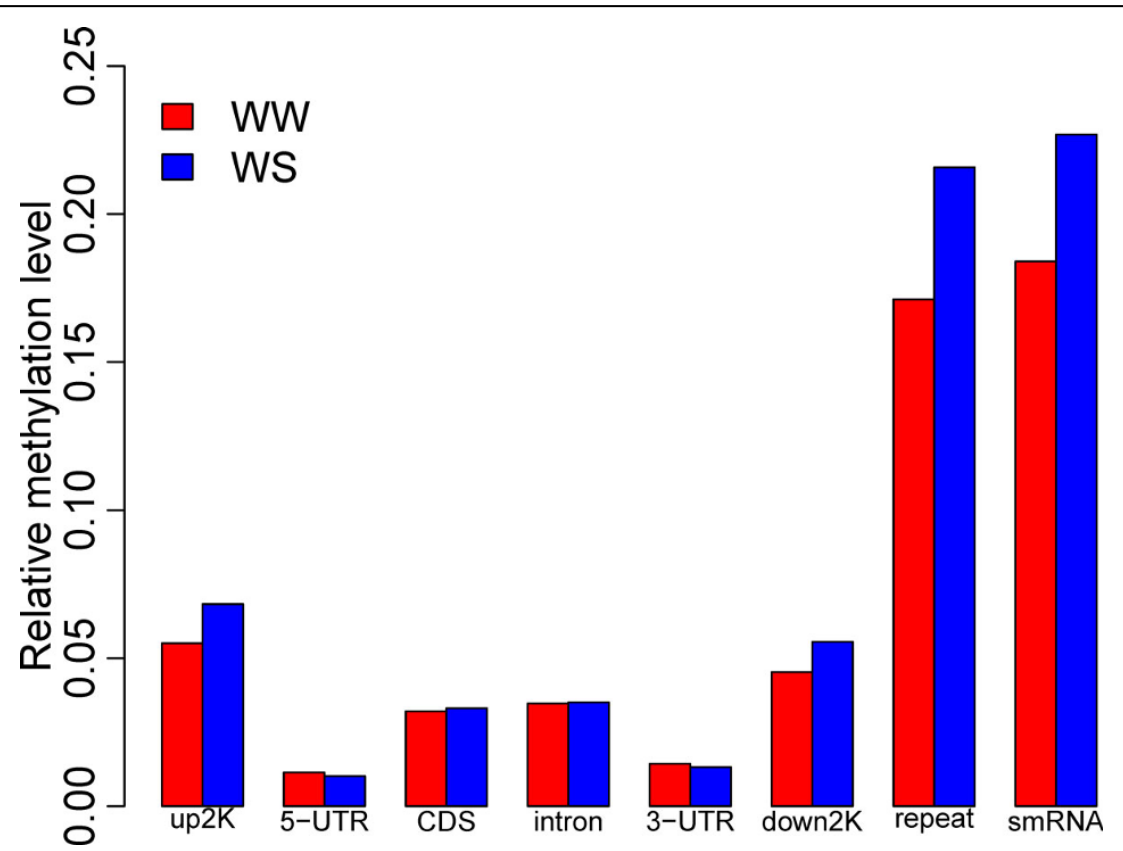

Figure 2 Relative methylation levels of the corresponding genomic region. The $y$ axis shows the relative methylation level in each element of genomic region ( $x$ axis), which contains upstream, 5' UTR, CDS, Intron, 3' UTR, Downstream and repeative sequences. 


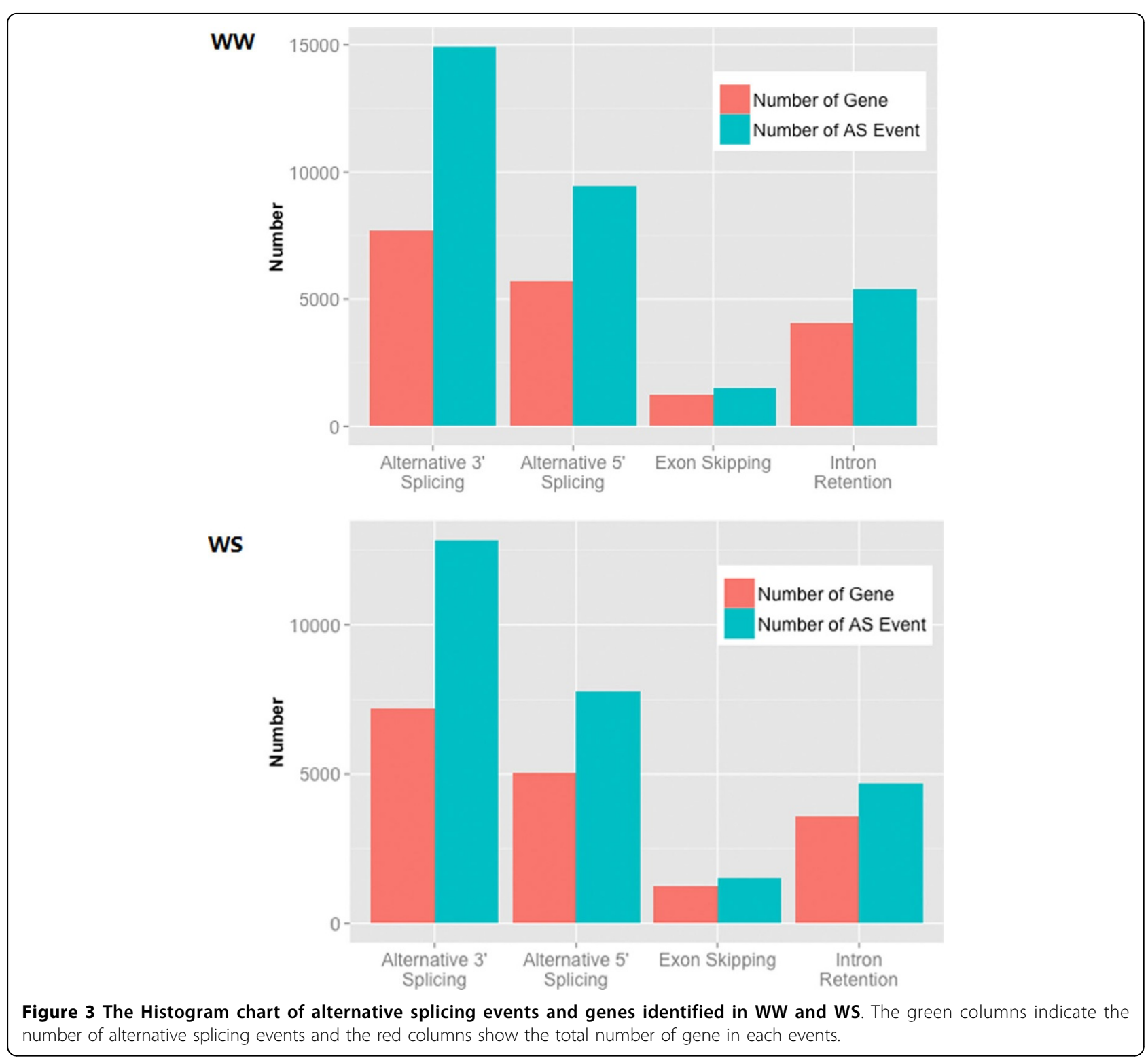

aligned to more than one location were discarded. Junction sequences were obtained by combining the exons of candidate genes. Junction reads and PE reads having only one end were compared to reference genes (5-bp overlap at least on the fusion point), and the candidate genes were supported by both PE reads and junction reads. The homologous candidate genes were detected and excluded, and the identification of alternative splicing sites was performed as described previously [24].

Collected potential splice sites by enumerating all possible pairs of donor sites (GT on the forward strand and $A C$ on the reverse strand) and acceptor sites (AG on the forward strand and CT on the reverse strand) inside intron regions. Secondly, filtered all potential splice sites through the information of supported reads. At least two unique mapped reads convered on the junction site and having a minimum of five bases on both sides of the junction. Finally, categorized all these splice sites into seven types including exon skipping (ES), intron retention (IR), alternative 5' splice site (A5SS), alternative 3' splice site (A3SS), mutually exclusive exons (MXE), alternative first exons (AFE), and alternative last exons (ALE).

\section{Gene ontology (GO) analysis}

$\mathrm{GO}$ annotations of poplar genes were downloaded from (http://bioinfo.cau.edu.cn/agriGO/download/item2term_46). GO comparative analyses between interested gene groups were performed using BGI WEGO (http://wego.genomics. org.cn/cgi-bin/wego/index.pl). GO enrichment analysis was 


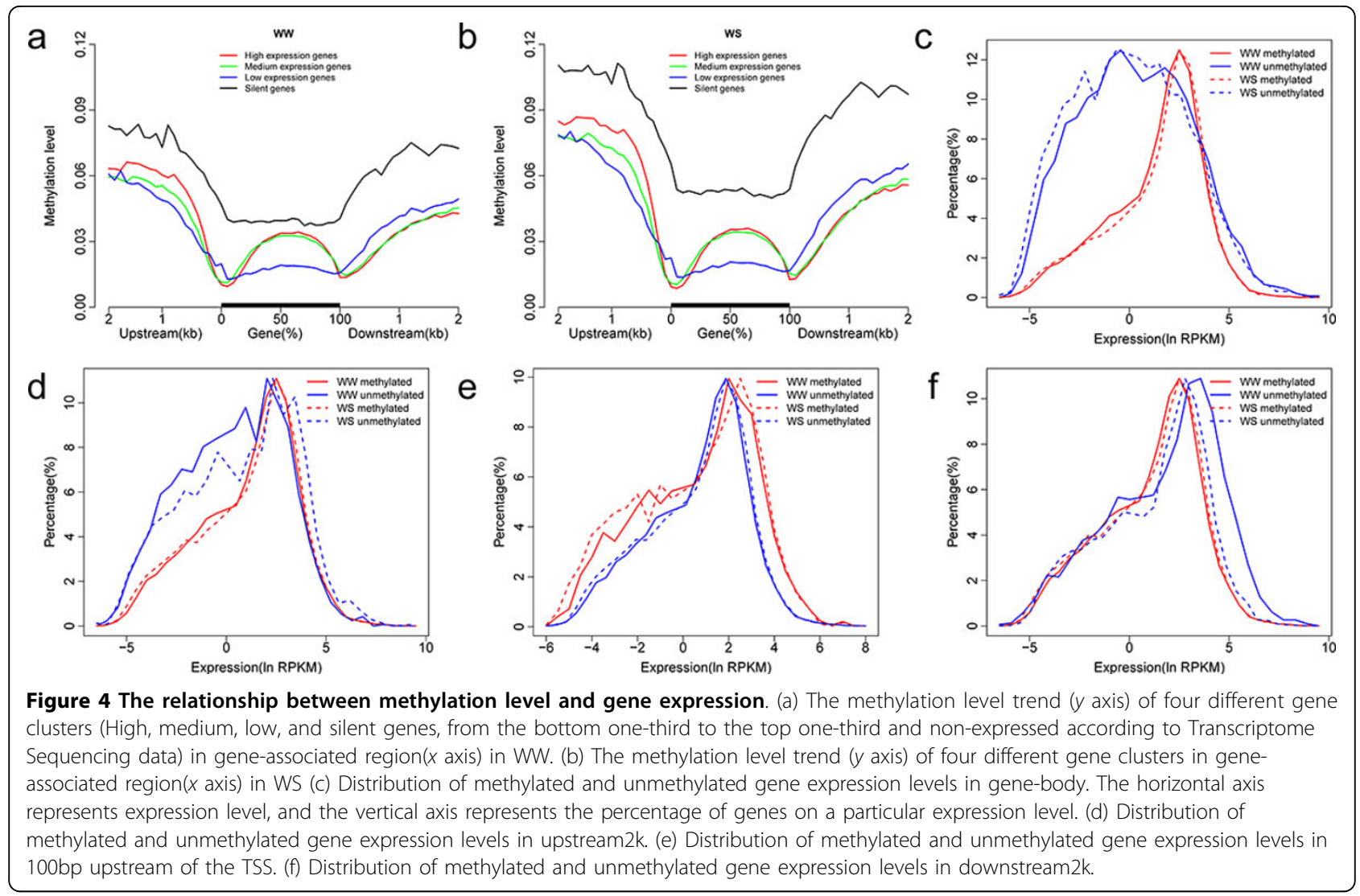

performed using Blast2GO and agriGO (http://bioinfo.cau. edu.cn/agriGO/) with Fisher's exact test [25].

\section{Additional material}

Additional file 1: Description of the data for the Populus of two treatments

Additional file 2: Three methylation patterns of Populus

Additional file 3: Seqlogo of the sequences proximal to DNA methylation cytosines. One stack for each position in these two sequence contexts $(\mathrm{CHG}, \mathrm{CH})$, The overall height of the stack indicates the sequence conservation at that position, while the height of bases within the stack indicates the relative frequency of each base at that position. (red $=\mathrm{T}$, green $=\mathrm{A}$, blue $=\mathrm{C}$, yellow $=\mathrm{G}$ )

Additional file 4: Density of methylcytosines identified in each chromosome in Populus. Red dots indicate the density of all methylcytosines in $10 \mathrm{~kb}$ windows. The top panel shows the Watson strand information and the bottom panel displays crick strand information.

Additional file 5: GO enrichment analysis newly methylated and demethylated genes

Additional file 6: Description of the data for the Populus of two treatments by Transcriptome Sequencing

Additional file 7: Results of two splicing forms. (a) Four kinds of alternative splicing types were compared to each other on methylation level; (b) the PCR result of the fusion genes verification.

Additional file 8: The correlation between gene methylation and gene expression. The upstream, gene body and downstream were split into 20 bins that lay on $\mathrm{x}$-axis for investigating the spearman rank correlation(y-axis) between methylation and expression. Red line stands for WW and blue line stands for WS.

Additional file 9: Category of co-regulated transcription factors Additional file 10: Category of transcription factors with methylated TE that located in promoter

Additional file 11: Category of transcription factors with methylated TE that located in gene

\section{Competing interests}

The authors declare that they have no competing interests.

\section{Authors' contributions}

DL PS designed and conducted the experiments, DL HL CH PS ZZ ST analyzed the data, DL HLPS WY XX drafted the manuscript, WY XX supervised the project. All authors have read and approved the final version of this manuscript.

\section{Declarations}

Publication charges for this work were supported by the National Natural Science Foundation of China $(31070597,31270656)$, the fund from the Ministry of Science and Technology of China (2009CB119101), and the Scientific Research and Graduate Training Joint Programs from BMEC (Stress Tolerance and DNA Methylation in Populus).

This article has been published as part of BMC Genetics Volume 15 Supplement 1, 2014: Selected articles from the International Symposium on Quantitative Genetics and Genomics of Woody Plants. The full contents of the supplement are available online at http://www.biomedcentral.com/ bmcgenet/supplements/15/S1. 


\section{Authors' details}

${ }^{1}$ College of Biological Sciences and Technology, National Engineering Laboratory for Tree Breeding, Beijing Forestry University, Beijing 100083, China. ${ }^{2} \mathrm{BGI}$-Shenzhen, Building 11, Beishan Industrial Zone, Yantian District, Shenzhen, Guangdong, China. ${ }^{3}$ BGI-Tianjin, E3 building, Airport Business Park, Tianjin Airport Economic Area,Tianjin, China.

Published: 20 June 2014

\section{References}

1. Jansson S, Douglas CJ: Populus: a model system for plant biology. Annu Rev Plant Biol 2007, 58:435-458,

2. Tuskan GA, Difazio S, Jansson S, Bohlmann J, Grigoriev I, Hellsten U, Putnam N, Ralph S, Rombauts S, Salamov A, et al: The genome of black cottonwood, Populus trichocarpa (Torr. \& Gray). Science 2006, 313(5793):1596-1604.

3. Tuskan GA: Short-rotation woody crop supply systems in the United States: what do we know and what do we need to know? Biomass and Bioenergy 1998, 14(4):307-315.

4. Bogeat-Triboulot MB, Brosche M, Renaut J, Jouve L, Le Thiec D, Fayyaz P, Vinocur B, Witters E, Laukens K, Teichmann T, et al: Gradual soil water depletion results in reversible changes of gene expression, protein profiles, ecophysiology, and growth performance in Populus euphratica, a poplar growing in arid regions. Plant physiology 2007, 143(2):876-892.

5. Tang S, Liang H, Yan D, Zhao Y, Han X, Carlson J, Xia X, Yin W: Populus euphratica: the transcriptomic response to drought stress. Plant Mol Biol 2013, 1-19.

6. Shuai $P$, Liang D, Zhang Z, Yin W, Xia X: Identification of droughtresponsive and novel Populus trichocarpa microRNAs by highthroughput sequencing and their targets using degradome analysis. Bmc Genomics 2013, 14(1):233.

7. Lister R, O'Malley RC, Tonti-Filippini J, Gregory BD, Berry CC, Millar AH, Ecker JR: Highly integrated single-base resolution maps of the epigenome in Arabidopsis. Cell 2008, 133(3):523-536.

8. Wang X, Elling AA, Li X, Li N, Peng Z, He G, Sun H, Qi Y, Liu XS, Deng XW: Genome-Wide and Organ-Specific Landscapes of Epigenetic Modifications and Their Relationships to mRNA and Small RNA Transcriptomes in Maize. The Plant Cell Online 2009, 21(4):1053-1069.

9. Lister R, Pelizzola M, Dowen RH, Hawkins RD, Hon G, Tonti-Filippini J, Nery JR, Lee L, Ye Z, Ngo QM, et al: Human DNA methylomes at base resolution show widespread epigenomic differences. Nature 2009, 462(7271):315-322.

10. Xiang H, Zhu J, Chen Q, Dai F, Li X, Li M, Zhang H, Zhang G, Li D, Dong Y, et al: Single base-resolution methylome of the silkworm reveals a sparse epigenomic map. Nature biotechnology 2010, 28(5):516-520.

11. Cao X, Jacobsen SE: Locus-specific control of asymmetric and CpNpG methylation by the DRM and CMT3 methyltransferase genes. Proceedings of the National Academy of Sciences 2002, 99(Suppl 4):16491-16498.

12. Razin A: CpG methylation, chromatin structure and gene silencing-a three-way connection. The EMBO journal 1998, 17(17):4905-4908.

13. Curradi M, Izzo A, Badaracco G, Landsberger N: Molecular mechanisms of gene silencing mediated by DNA methylation. Molecular and cellular biology 2002, 22(9):3157-3173.

14. Uthup TK, Ravindran M, Bini K, Thakurdas S: Divergent DNA Methylation Patterns Associated with Abiotic Stress in Hevea brasiliensis. Molecular Plant 2011, 4(6):996-1013.

15. Labra M, Ghiani A, Citterio S, Sgorbati S, Sala F, Vannini C, RuffiniCastiglione M, Bracale M: Analysis of Cytosine Methylation Pattern in Response to Water Deficit in Pea Root Tips. Plant Biology 2002, 4(6):694-699.

16. Colaneri AC, Jones AM: Genome-Wide Quantitative Identification of DNA Differentially Methylated Sites in Arabidopsis Seedlings Growing at Different Water Potential. PloS one 2013, 8(4):e59878.

17. Wang W-S, Pan Y-J, Zhao X-Q, Dwivedi D, Zhu L-H, Ali J, Fu B-Y, Li Z-K: Drought-induced site-specific DNA methylation and its association with drought tolerance in rice (Oryza sativa L.). Journal of experimental botany 2010.

18. Yu Y, Yang $X$, Wang $H$, Shi F, Liu Y, Liu J, Li L, Wang D, Liu B: Cytosine Methylation Alteration in Natural Populations of Leymus chinensis Induced by Multiple Abiotic Stresses. PloS one 2013, 8(2): e55772.
19. Rico L, Ogaya R, Barbeta A, Peñuelas J: Changes in DNA methylation fingerprint of Quercus ilex trees in response to experimental field drought simulating projected climate change. Plant Biol (Stuttg) 2013, 16(2):419-427.

20. Griffiths H, Parry MA: Plant responses to water stress. Annals of Botany 2002, 89: 801-802.

21. Xu X, Yang F, Xiao X, Zhang S, Korpelainen H, Li C: Sex-specific responses of Populus cathayana to drought and elevated temperatures. Plant, cell \& environment 2008, 31(6):850-860.

22. Regier N, Streb S, Cocozza C, Schaub M, Cherubini P, Zeeman SC, Frey B: Drought tolerance of two black poplar (Populus nigra L.) clones: contribution of carbohydrates and oxidative stress defence. Plant, cell \& environment 2009, 32(12):1724-1736.

23. Hayatsu H, Tsuji K, Negishi K: Does urea promote the bisulfite-mediated deamination of cytosine in DNA? Investigation aiming at speeding-up the procedure for DNA methylation analysis. Nucleic Acids Symposium Series 2006, 50(1):69-70.

24. Zhang GJ, Guo GW, Hu XD, Zhang Y, Li QY, Li RQ, Zhuang RH, Lu ZK, He ZQ, Fang XD: Deep RNA sequencing at single base-pair resolution reveals high complexity of the rice transcriptome. Genome research 2010, 20(5):646-654.

25. Du Z, Zhou X, Ling Y, Zhang Z, Su Z: agriGO: a GO analysis toolkit for the agricultural community. Nucleic acids research 2010, 38(suppl 2):W64-W70.

26. Horiuchi T, Aigaki T: Alternative trans-splicing: a novel mode of premRNA processing. Biology of the Cell 2006, 98(2):135-140.

27. Akiva P, Toporik A, Edelheit S, Peretz Y, Diber A, Shemesh R, Novik A, Sorek $\mathrm{R}$ : Transcription-mediated gene fusion in the human genome. Genome research 2006, 16(1):30-36.

28. Filichkin SA, Priest HD, Givan SA, Shen R, Bryant DW, Fox SE, Wong WK, Mockler TC: Genome-wide mapping of alternative splicing in Arabidopsis thaliana. Genome research 2010, 20(1):45-58.

29. Mazzucotelli E, Mastrangelo AA, Crosatti C, Guerra D, Stanca AM, Cattivelli L: Abiotic stress response in plants: When post-transcriptional and posttranslational regulations control transcription. Plant Sci 2008, 174(4):420-431.

30. Palusa SG, Ali GS, Reddy ASN: Alternative splicing of pre-mRNAs of Arabidopsis serine/arginine-rich proteins: regulation by hormones and stresses. The Plant Journal 2007, 49(6):1091-1107.

31. Li X, Wang X, He K, Ma Y, Su N, He H, Stolc V, Tongprasit W, Jin W, Jiang J: High-Resolution Mapping of Epigenetic Modifications of the Rice Genome Uncovers Interplay between DNA Methylation, Histone Methylation, and Gene Expression. The Plant Cell Online 2008, 20(2):259-276.

32. Zemach A, McDaniel IE, Silva P, Zilberman D: Genome-wide evolutionary analysis of eukaryotic DNA methylation. Science 2010, 328(5980):916-919.

33. Perez-Rodriguez P, Riano-Pachon DM, Correa LG, Rensing SA, Kersten B, Mueller-Roeber B: PInTFDB: updated content and new features of the plant transcription factor database. Nucleic acids research 2010, 38(Database):D822-827.

34. Thornburg BG, Gotea V, Makalowski W: Transposable elements as a significant source of transcription regulating signals. Gene 2006, 365:104-110.

35. Vining KJ, Pomraning KR, Wilhelm $L$, Priest HD, Pellegrini M, Mockler TC, Freitag M, Strauss SH: Dynamic DNA cytosine methylation in the Populus trichocarpa genome: tissue-level variation and relationship to gene expression. Bmc Genomics 2012, 13:27.

36. Li XY, Wang XF, He K, Ma YQ, Su N, He H, Stolc V, Tongprasit W, Jin WW, Jiang JM, et al: High-resolution mapping of epigenetic modifications of the rice genome uncovers interplay between DNA methylation, histone methylation, and gene expression. Plant Cell 2008, 20(2):259-276.

37. Shukla S, Kavak E, Gregory M, Imashimizu M, Shutinoski B, Kashlev M, Oberdoerffer P, Sandberg R, Oberdoerffer S: CTCF-promoted RNA polymerase II pausing links DNA methylation to splicing. Nature 2011, 479(7371):74-79.

38. Chodavarapu RK, Feng SH, Bernatavichute YV, Chen PY, Stroud H, Yu YC Hetzel JA, Kuo F, Kim J, Cokus SJ, et al: Relationship between nucleosome positioning and DNA methylation. Nature 2010, 466(7304):388-392.

39. Zhang X, Yazaki J, Sundaresan A, Cokus S, Chan SW, Chen H, Henderson IR, Shinn $P$, Pellegrini $M$, Jacobsen $S E$, et al: Genome-wide high-resolution mapping and functional analysis of DNA methylation in arabidopsis. Cell 2006, 126(6):1189-1201. 
40. Li X, Zhu J, Hu F, Ge S, Ye M, Xiang H, Zhang G, Zheng X, Zhang H, Zhang $S$, et al: Single-base resolution maps of cultivated and wild rice methylomes and regulatory roles of DNA methylation in plant gene expression. Bmc Genomics 2012, 13:300.

41. Kapitonov W, Jurka J: A universal classification of eukaryotic transposable elements implemented in Repbase. Nat Rev Genet 2008, 9(5):411-412.

42. Schwartz D, Dennis E: Transposase activity of the Ac controlling element in maize is regulated by its degree of methylation. Mol Gen Genet 1986, 205(3):476-482.

43. Banks JA, Masson P, Fedoroff $\mathrm{N}$ : Molecular mechanisms in the developmental regulation of the maize Suppressor-mutator transposable element. Genes \& Development 1988, 2(11):1364-1380.

44. Martienssen RA, Colot V: DNA methylation and epigenetic inheritance in plants and filamentous fungi. Science 2001, 293(5532):1070-1074

45. Kato M, Miura A, Bender J, Jacobsen SE, Kakutani T: Role of CG and nonCG methylation in immobilization of transposons in Arabidopsis. Current Biology 2003, 13(5):421-426.

46. Bourque $\mathrm{G}$, Leong $\mathrm{B}$, Vega VB, Chen $\mathrm{X}$, Lee $\mathrm{YL}$, Srinivasan $\mathrm{KG}$, Chew IL, Ruan $Y$, Wei $\mathrm{CL}, \mathrm{Ng} \mathrm{HH}$, et al: Evolution of the mammalian transcription factor binding repertoire via transposable elements. Genome research 2008, 18(11):1752-1762

doi:10.1186/1471-2156-15-S1-S9

Cite this article as: Liang et al:: Single-base-resolution methylomes of populus trichocarpa reveal the association between DNA methylation and drought stress. BMC Genetics 2014 15(Suppl 1):S9.

\section{Submit your next manuscript to BioMed Central} and take full advantage of:

- Convenient online submission

- Thorough peer review

- No space constraints or color figure charges

- Immediate publication on acceptance

- Inclusion in PubMed, CAS, Scopus and Google Scholar

- Research which is freely available for redistribution

Submit your manuscript at www.biomedcentral.com/submit
C Biomed Central 